\title{
Immunopathologia Persa
}

\section{Controversy with bromhexine in COVID-19; where we stand}

\author{
Ramin Tolouian $^{1 * \mathbb{(}}$, Zuber D. Mulla ${ }^{\mathbf{D}^{\circledR}}$ \\ ${ }^{1}$ Division of Nephrology, University of Arizona, Tucson, AZ, USA \\ ${ }^{2}$ Department of Obstetrics and Gynecology, Texas Tech University Health Sciences Center El Paso, El Paso, TX, USA, and Julia Jones \\ Matthews Department of Public Health, Texas Tech University Health Sciences Center, Lubbock, TX, USA
}

*Correspondence to

Prof. Ramin Tolouian, Email;

Ramin.tolouian@va.gov

Received 1 Aug. 2020

Accepted 8 Aug. 2020

Published online 12 Aug. 2020

\author{
Key point \\ It is possible that bromhexine hydrochloride, by inhibiting TMPRSS2, can prevent the priming of COVID-19 and \\ abort the cell entry of the SARS-CoV-2. \\ Keywords: Bromhexine, COVID-19, TMPRSS2, SARS-CoV-2
}

Citation: Tolouian R Mulla ZD. Controversy with bromhexine in COVID-19; where we stand. Immunopathol Persa. $2021 ; 7(2): \mathrm{e} 12$. DOI:10.34172/ ipp.2021.12.
$\mathrm{I}$ n December 2019, Covid-19 was reported for the first time in China. Shortly after, in March 2020, it became a pandemic. While the majority of countries are experiencing the end of the first wave of the disease and have started to remove the social restriction orders, the second wave of the virus infection has begun to rise in some countries, causing them to again increase restrictions.

COVID-19 is a significant public health issue and has had a significant global socioeconomic impact. According to the Johns Hopkins University Coronavirus Resource Center as of August 10 2020 , the number of deaths in the world has surpassed 735,000 and the number is increasing on a daily basis (1).

Over the past 8 months, multiple medications and therapeutic options have been tried. No effective medication has been introduced and vaccines are still undergoing clinical testing.

Bromhexine is an over-the-counter, safe and inexpensive mucolytic that has been used in clinical medicine since 1963. Its main application has been to improve mucociliary clearance in the pulmonary system. Bromhexine is able to inhibit serine protease [transmembrane serine protease 2 (TMPRSS2)] and, via this mechanism, has been used to successfully treat distant metastasis in mice models with prostate cancer. Later, its role in prevention of influenza infection by inhibition of TMPRSS2 has been observed. With the same rationale, it had been suggested, that bromhexine hydrochloride, by inhibiting TMPRSS2, can prevent the priming of COVID-19 and abort the cell entry of the virus. Therefore, it should be considered a potential treatment to prevent COVID-19 infection (2-4).

Recently, Ansarin et al reported the results of a randomized clinical trial that evaluated the effect of Bromhexine on clinical outcomes and mortality in hospitalized patients with COVID-19.

In this clinical trial, 78 patients were randomized into two groups. Both groups were on low dose $200 \mathrm{mg} /$ day hydroxychloroquine (HCQ). The treatment group, in addition to HCQ, received bromhexine $8 \mathrm{mg}$ TID. The ICU admissions ( 2 out of 39 versus 11 out of $39, P=0.006$ ), intubation ( 1 out of 39 vs. 9 out of 39 , $P=0.007)$ and death ( 0 out of 39 vs. 5 out of $39, P=0.027$ ), were all significantly lower in the bromhexine group.

The study demonstrated a tremendous advantage of bromhexine for mortality and morbidity.

It was also observed that cardinal respiratory symptoms, such as cough and dyspnea, were remarkably less after a twoweek treatment with bromhexine. Qualitative C-reactive protein $(\mathrm{CRP})$ in all of the patients who received bromhexine after two weeks improved and turned negative, while in the control group, $83 \%$ still had positive CRP.

The authors concluded that administration 
of bromhexine in the early stage of the disease is safe, bromhexine is an affordable medication and a game changer (5). This trial had many limitations. The main limitations were a small sample size and being a single center report. The study, while randomized, was an open label trial. Study subjects who are aware of their treatment allocation may differ from blinded subjects in how they report their symptoms. Additionally, investigators in an open label trial may probe study patients differentially by treatment status thereby leading to observer bias (6).

This study is the first clinical trial that assessed the efficacy of bromhexine in COVID-19 and despite the limitations of the study, the results are very promising.

Whether this promising result is because of bromhexine alone or combination of low dose HCQ [200 mg/d] and bromhexine is not clear at this point.

Recently, Hörnich et al studied the interaction of S-protein in SARS-CoV-2 with the ACE2 (angiotensinconverting enzyme 2) receptor and TMPRSS2. Their paper has not been peer reviewed yet. They concluded that SARS-CoV-2 does not require TMPRSS2 on target cells for cell-cell fusion and suggested that bromhexine is not able to inhibit cell-to-cell fusion in COVID-19 infection. They also moved one step further and claimed that bromhexine may even moderately enhance fusion. Their experiment claimed that SARS-COV-2 fusion, as compared to the first SARS virus, depends more on ACE2 receptor expression rather than protease activation (7).

There is conflicting data about the role of bromhexine in the prevention and treatment of COVID-19. In clinical practice, the benefits of using bromhexine in COVID-19 far outweigh the risk for now. Further, high scale, welldesigned clinical trials are needed to shed light on this issue. There are at least three large scale clinical trials in China, Estonia and Mexico underway at this time.

Authors' contribution

$\mathrm{RT}$; concept, design and manuscript draft. ZDM conducted final revision. Both authors read and signed the final paper.

Conflicts of interest

The authors declare that there is no conflict of interest.

Ethical considerations

Ethical issues (including plagiarism, data fabrication, double publication) have been completely observed by the authors.

Funding/Support

None.

References

1. Johns Hopkins University Coronavirus Resource Center. Available from: https://coronavirus.jhu.edu/map.html.

2. Tolouian R, Tolouian AC, Ardalan M. Blocking serine protease (TMPRSS2) by Bromhexine; looking at potential treatment to prevent COVID-19 infection. Marshall Journal of Medicine. 2020;6(3):9. doi:10.33470/2379-9536.1286

3. Maggio R, Corsini GU. Repurposing the mucolytic cough suppressant and TMPRSS2 protease inhibitor bromhexine for the prevention and management of SARS-CoV-2 infection. Pharmacol Res. 2020;157:104837. doi: 10.1016/j. phrs.2020.104837

4. Depfenhart M, de Villiers D, Lemperle G, Meyer M, Di Somma S. Potential new treatment strategies for COVID-19: is there a role for bromhexine as add-on therapy? Intern Emerg Med. 2020 May 26:1-12. doi: 10.1007/s11739-020-02383-3.

5. Ansarin k, Tolouian R, Ardalan M, Taghizadeh A, Varshochi M, Teimouri S, et al. Effect of bromhexine on clinical outcomes and mortality in COVID-19 patients: A randomized clinical trial. Biolmpacts. 2020;10:209-15. doi: 10.34172/bi.2020.27

6. Hróbjartsson A, Emanuelsson F, Skou Thomsen AS, Hilden J, Brorson S. Bias due to lack of patient blinding in clinical trials. A systematic review of trials randomizing patients to blind and nonblind sub-studies. Int J Epidemiol. 2014;43(4):1272-83. doi: 10.1093/ije/dyu115.

7. Hörnich B, Großkopf A, Schlagowski S, Tenbusch M, Napiel $F$, Hahn A. SARS-CoV-2 differs from SARS-CoV in the requirements for receptor expression and proteolytic activation to trigger cell-cell fusion and is not inhibited by Bromhexine. bioRxiv. 2020. doi: 10.1101/2020.07.25.221135 